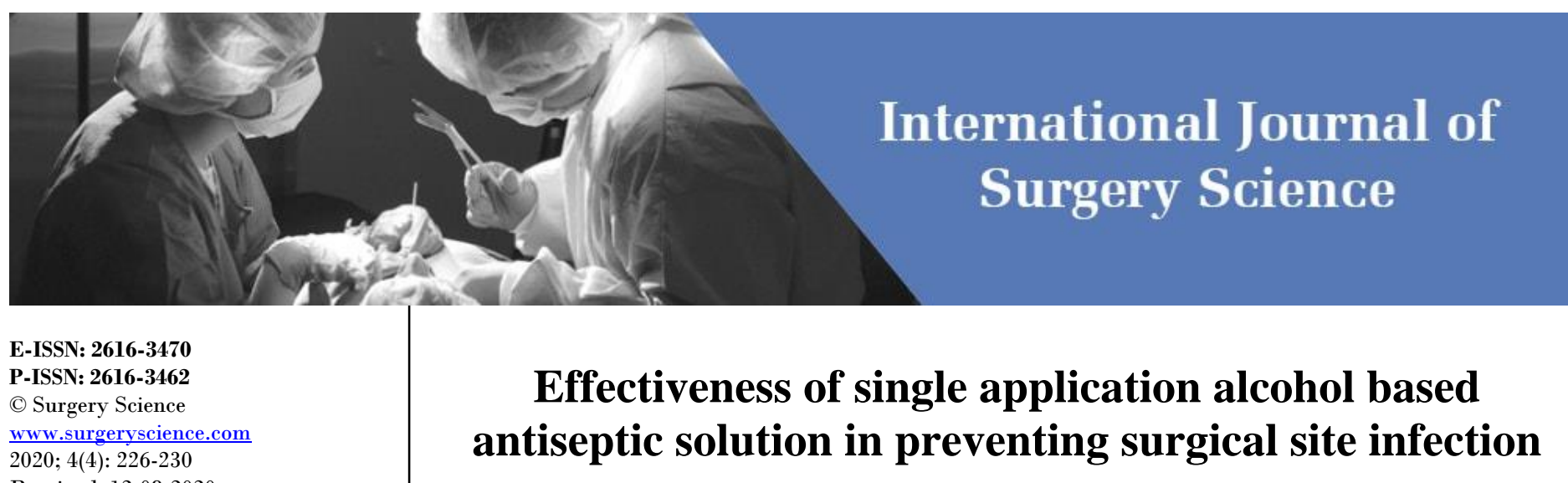

Received: $12-08-2020$

Accepted: 19-09-2020

Dr. Ashok Gopinath

Associate Professor, Department of General Surgery, Sree Mookambika Institute of Medical Sciences,

Kulasekharam, Kanyakumari,

Tamil Nadu, India

\section{Karthick MP}

Junior Resident, Department of General Surgery, Sree Mookambika Institute of Medical Sciences, Kulasekharam, Kanyakumari, Tamil Nadu, India

\section{Aswathy Harikumar}

Junior Resident, Department of

General Surgery, Sree Mookambika

Institute of Medical Sciences,

Kulasekharam, Kanyakumari,

Tamil Nadu, India

\section{Anandaraj A}

Junior Resident, Department of General Surgery, Sree Mookambika Institute of Medical Sciences,

Kulasekharam, Kanyakumari,

Tamil Nadu, India

\section{Corresponding Author:} Dr. Ashok Gopinath

Associate Professor, Department of General Surgery, Sree Mookambika Institute of Medical Sciences,

Kulasekharam, Kanyakumari,

Tamil Nadu, India

\section{Dr. Ashok Gopinath, Karthick MP, Aswathy Harikumar and Anandaraj A}

DOI: https://doi.org/10.33545/surgery.2020.v4.i4d.563

\section{Abstract}

Background: Surgical site infections are the most commonly encountered problem in all surgical procedure. The main aim of the study is finding out the effectiveness of single application alcohol based antiseptic solution during preoperative preparation to reduce the surgical site infections.

Materials and Methods: It is a prospective study of patients who underwent major and minor procedure in the duration of August 2019 - December 2019. The primary outcome of the study was evaluated on the basis of type of procedure, days of hospital stay, postoperative complications like seroma and pus discharge.

Results: Out of two hundred patients included in this study, 100 patients underwent minor procedure and the other 100 underwent major procedure. Age distribution for minor cases less than 25 is 3, 26-50 is 42 and 51-75 is 103 with mean $\pm \mathrm{SD}$ is $50.41 \pm 14.25$ and for major cases less than 25 is 1, 26-50 is $51,51-75$ is 48 with mean $\pm \mathrm{SD}$ is $50.87 \pm 13.59$. Sex distribution of minor cases male -48 \& female -52 , major cases with distribution of male -45 and female -55 . Hospitalisation of all minor cases are less than 1 day with mean value of 1 and major cases less than 1 day are 4, 2-3 days are 86 and more than 3 days are 10 with mean value of 2.3. Each patient was carefully monitored for the period of 30 days from the day of surgery and the complications, if any was treated accordingly. Out of 100 minor cases, seroma formation was noted in 3 patients and pus discharge in 4 patients. Collections were evacuated thorough wound wash and saline dressing were done (no antibiotics were given for these cases both preoperative as well as postoperative period).Whereas, Out of 100 major cases (single dose of preoperative antibiotics are given) seroma formation were noted in 7 patients, seroma collections were evacuated, thorough wound wash and saline dressing were done (no antibiotics were given for these cases), Pus discharge was found in 7 patients, among them 4 were treated with I V Antibiotics for 5 days according to pus culture and sensitivity report. Conclusion: The 200 patients who underwent procedure with single application of alcohol based antiseptic solution ( $2 \%$ chlorhexidine) for pre-operative preparation, was found to be effective against surgical site infections.

Keywords: Chlorhexidine digluconate, surgical site infection, preoperative preparation, postsurgical infection management

\section{Introduction}

Any infection or collection near the site of surgical incision within 30 days of procedure is named as Surgical Site Infection (SSI); it is the third most common infection \& nearly accounts for $15 \%$ of all nosocomial infections which directly contribute to the surgical mortality and morbidity each year worldwide ${ }^{[1]}$. The CDC has developed the set of standard criteria in management of surgical site infections. In an attempt to understand the mode of spread and to calculate the surveillance rate of SSI occurring after the successful procedure. They are broadly classified into three types: A) Superficial incisional SSI which involves only the skin and subcutaneous layer. B) Deep incisional SSI which involves skin, subcutaneous layer along with the fascia. C) Organ /space SSI which involves any other part of anatomy which is open. The most common source of SSI in majority of cases is the native patient's flora skin and visceral organs ${ }^{[2]}$. Staphylococcus, a gram positive aerobic cocci are the most common organism involved in SSI other organism includes E.coli, Bacillus, and Yeast ${ }^{[3]}$. When skin is incised, underlying tissue is exposed to overlying endogenous flora which leads to the formation of surgical site infections ${ }^{[4]}$. The first and foremost step needed to reduce the surgical site infection is by providing appropriate preoperative antibiotics, use of effective preoperative preparation technique. 


\section{Materials and Methods}

In this study, 200 patients were selected who visited our hospital in the period of August 2019 - November 2019. A prospective study analysis is made for all two hundred cases on the basis of types of illness, type of procedure, hospital stay and postoperative complications. All the patients who gave consent for this study are subjected to preoperative evaluation and they were categorised on the basis of diagnosis as major and minor groups. Minor group were treated on outpatient (day-care) basis with hospital stay less than one day, with nil preoperative and post-operative antibiotics. After successful procedure each patient was managed with analgesics alone (T. Paracetamol 500 $\mathrm{MG})$. Major group were treated as inpatients with hospital stay more than 1 day, and were given a single dose of pre-operative antibiotics 30 mins prior surgery. The same protocol was followed for all the patients and their response was analysed by Statistical analysis was done using SPSS 23.0.

\section{Results}

Out of two hundred patients included in this study, 100 patients underwent minor procedure and the other 100 underwent major procedure. Age distribution for minor cases less than 25 is 3, 2650 is 42 and 51-75 is 103 with mean \pm SD is $50.41 \pm 14.25$ and for major cases less than 25 is $1,26-50$ is $51,51-75$ is 48 with mean \pm SD is $50.87 \pm 13.59$ (Graph-1). Out of 200 patients, Sex distribution of minor cases male - $48 \&$ female -52 , major cases with distribution of male -45 and female -55 . Comparing male patients females were at higher distribution rate in both minor and major patients (Table-1). Out of all 200 cases, all 100 minor cases are treated as day care procedure, every case is discharged in less than 1 day and Out of 100 major cases, 4 patients are discharged in less than 4 days, 86 patients in 2-3 days and 10 cases in more than 3 days. All minor procedures patients are subjected to preoperative evaluation and procedure were done under local anaesthesia, Out of 100 cases, incision and drainage was done in majority which accounts for 49 cases, 36 excision and biopsy, 8 disarticulations, 3 incisional biopsy, 2 nail removals, 2 trucut biopsy (Graph-2, 3). Out of 100 cases in major category, modified radical mastectomy and mesh repair accounts for 13 cases, total thyroidectomy 12, laparoscopic cholecystectomy 11 , haemorrhoidectomy 9 , inguinal hernia 8 , excision and biopsy 7 , lateral sphinctorotomy 2, amputation 7, appendectomy 2, Webster procedure, other procedure 8 . Out of all 100 cases in minor procedure, 3 patient presented with seroma formation and 4 patient presented with pus discharge, collections and discharge are evacuated and they are treated conservatively no antibiotics were given to them. Out of 100 cases in major cases, seroma was noted in 7 cases and pus discharge in 7 patients these cases are treated conservatively with thorough saline wash and daily saline dressing were given. Out of 7 pus discharge cases, 4 patients were treated with I V Antibiotics for 5 days according to pus culture and sensitivity report (Table-2 and Graph-3, 4)

\section{Discussion}

The cornerstone practises in reducing the danger of surgical site infection is by reducing the microbial skin burden on the operative site. A huge amount of research has addressed with methods aimed to prove chlorhexidine in view of effective in reducing the surgical site infections in surgical programs.
Maintaining normothermia as against mild hypothermia has been shown to decrease the SSI rate from $19 \%$ to $6 \%$ and to decrease hospital stay in patients ${ }^{[9]}$. In our analysis, we have studied the effectiveness in 200 patients which clearly states that the utilization of chlorhexidine -alcohol based antiseptic solution in preoperative period has found effective in reducing the SSI. Edmiston et al. (2010) ${ }^{[8]}$ chlorhexidine is a simple, effective in reducing the risk of SSIs. Single-dose of broadspectrum antibiotic alongside alcohol-based preparation within the perioperative period has been shown to scale back infection rates in surgical patients. Ishai Levin et al., (2011) [9] demonstrates that antisepsis with chlorhexidine and alcohol was related to a big reduction within the rate of SSIs compared to povidone-iodine antisepsis in patients undergoing elective gynaecological laparotomies. Culligan et al. ${ }^{[15]}$ demonstrated that cleansing with chlorhexidine as opposed to povidone-iodine reduced the rate of bacterial colonization in the operative field. Sowapat et al ${ }^{[16]}$ didnt find a big difference in patients undergoing total abdominal hysterectomy after cleansing with chlorhexidine or povidone-iodine solutions. Michael T Adler et al., (2012) ${ }^{[17]}$ made a study on 50 participants which shows $2 \%$ chlorhexidine - alcohol-based solution had excellent bactericidal efficacy and was superior to povidone-iodine for cleansing the maternal abdomen. Aaron et al., (2010) ${ }^{[7]}$ studied preoperative chlorhexidine preparation in 1134 patients among them 157 patients completely complaint to chlorhexidine preparation with an infection rate of $1.6 \%$ proves that chlorhexidine is that the better antiseptic solution to scale back infection. Charles E. Edmiston et al. ${ }^{[8]}$ clinical studies have shown chlorhexidine gluconate to be a secure and effective perioperative skinprepping agent. By employing a standardized protocol which ends up in high skin surface concentrations sufficient to inhibit/kill skin colonizing flora, including methicillin-resistant Staphylococcus aureus. Gulden Menderes et al., (2012) ${ }^{[10]}$ there's good evidence that $\mathrm{CHG}$-alcohol is superior to aqueous PVI - a crucial competitor - altogether three areas of skin antisepsis and he also states that perceived efficacy of $\mathrm{CHG}$ in skin antisepsis is usually actually supported evidence for the efficacy of the CHG-alcohol combination. Matthias Maiwald et al. ${ }^{[12]}$ perceived efficacy of $\mathrm{CHG}$ in skin antisepsis is usually actually supported evidence for the efficacy of the CHGalcohol combination. It's better in reducing the danger of surgical site infection.

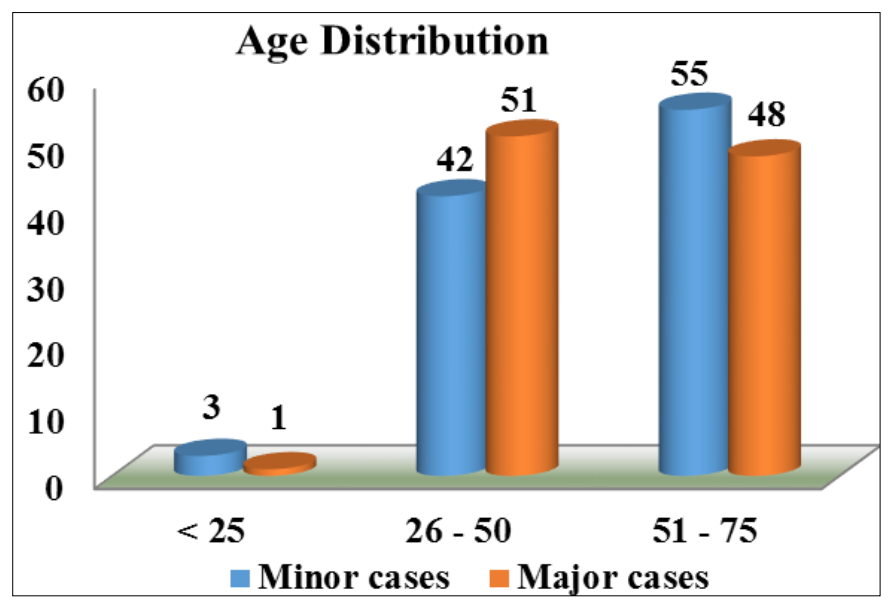

Graph 1: Distribution of patients based on the age 
Table 1: Distribution of patients based on the gender

\begin{tabular}{|c|c|c|c|c|}
\hline \multirow{2}{*}{ Gender } & \multicolumn{2}{|c|}{ Minor cases } & \multicolumn{2}{c|}{ Major cases } \\
\cline { 2 - 5 } & Number & Percentage (\%) & Number & Percentage (\%) \\
\hline Male & 48 & 48.00 & 45 & 45.00 \\
\hline Female & 52 & 52.00 & 55 & 55.00 \\
\hline Total & 100 & 100.00 & 100 & 100.00 \\
\hline
\end{tabular}

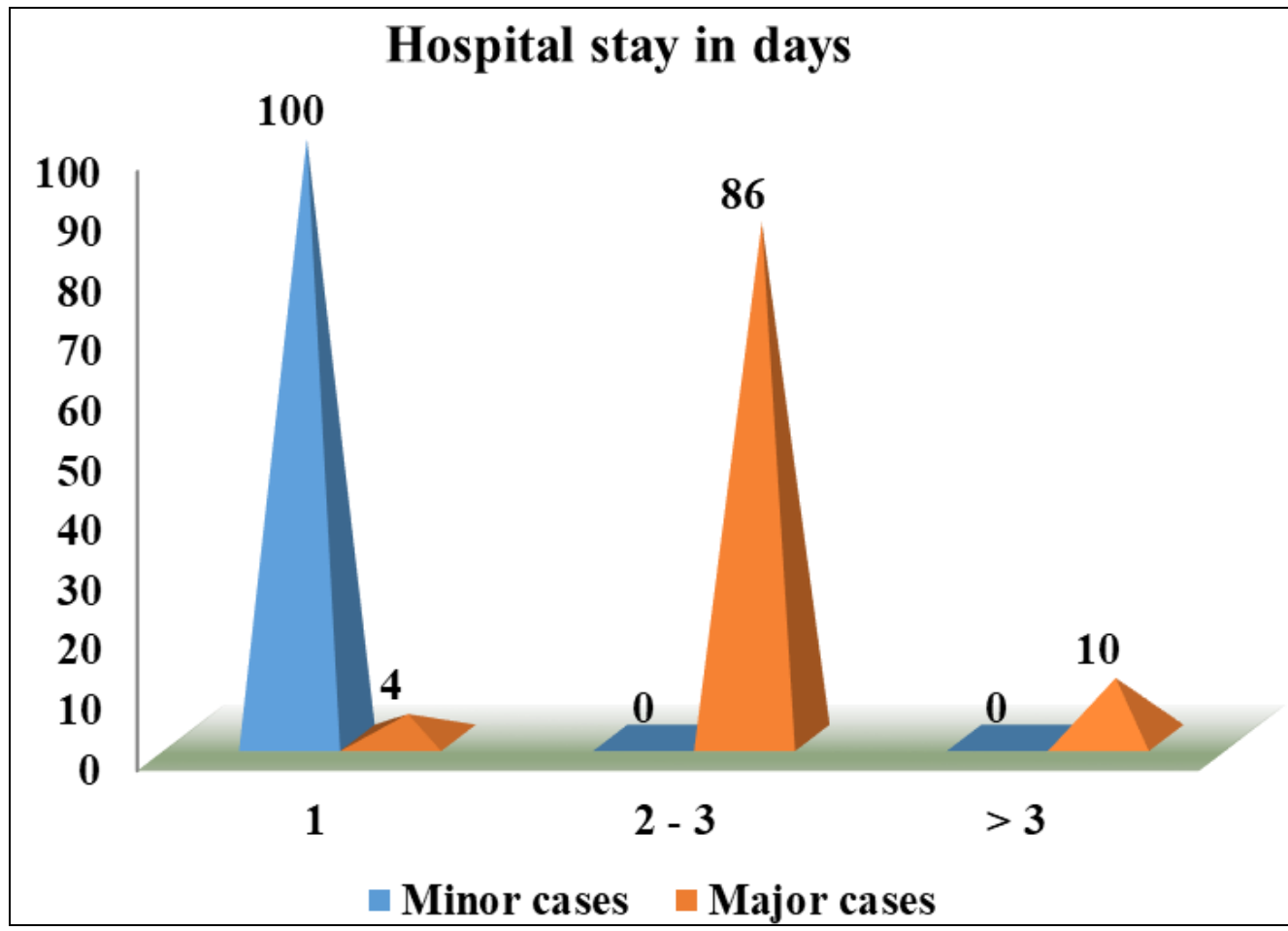

Graph 2: Distribution of patients based on the hospital stay

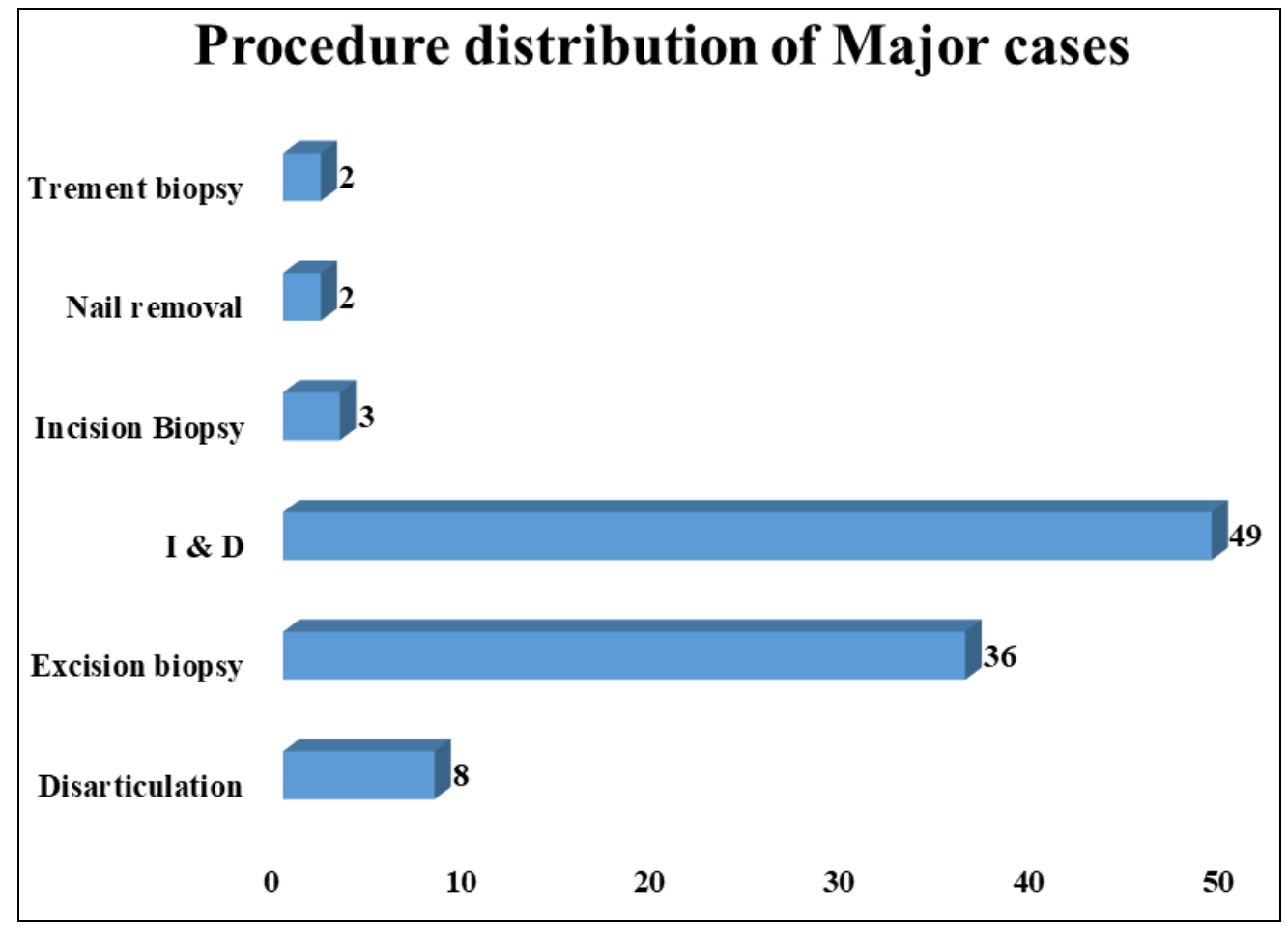

Graph 3: Distribution of patients based on the procedure 


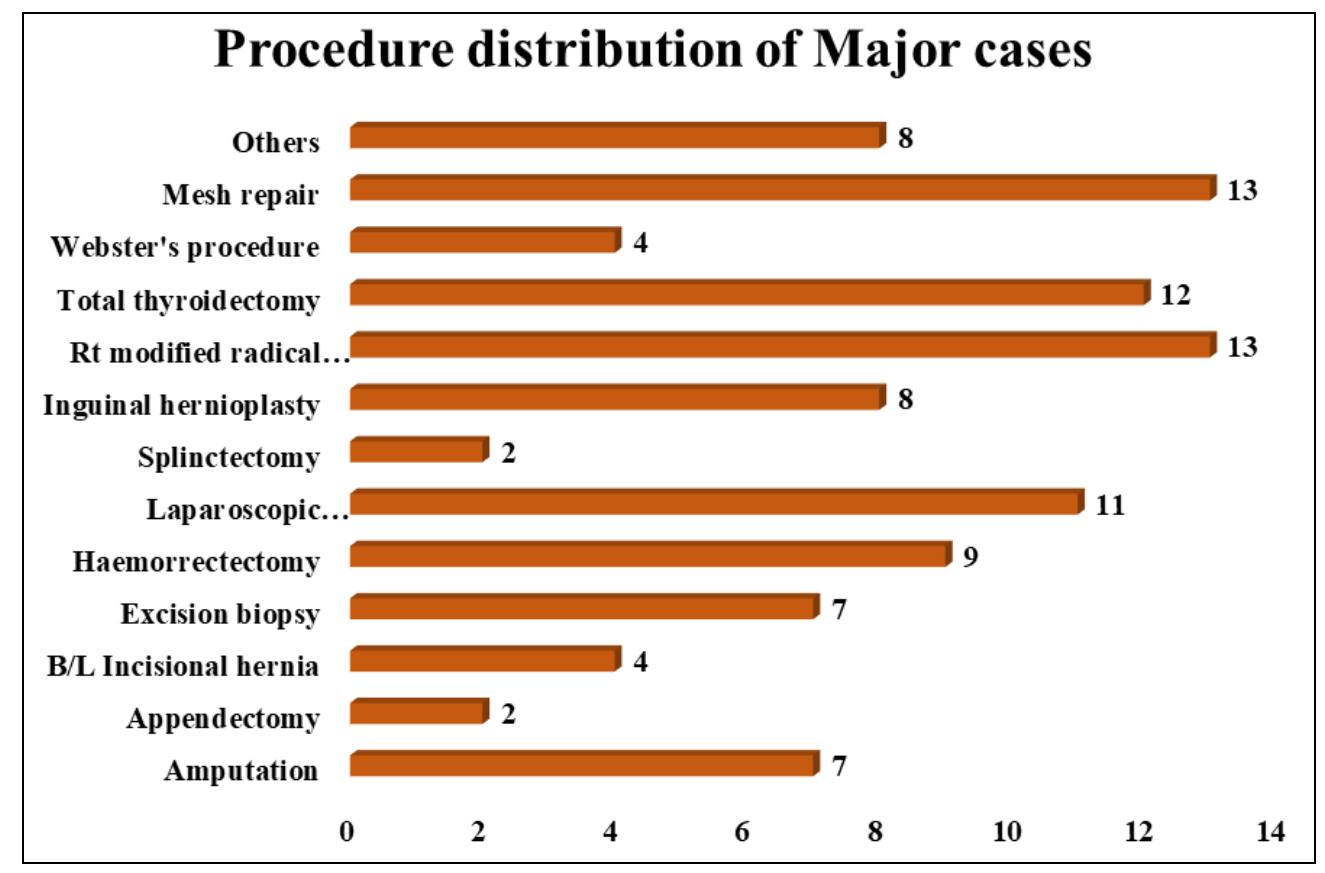

Graph 4: Distribution of patients based on the procedure

Table 2: Distribution of patients based on the complications

\begin{tabular}{|c|c|c|c|c|}
\hline \multirow{2}{*}{ Complications } & \multicolumn{2}{|c|}{ Minor cases } & \multicolumn{2}{c|}{ Major cases } \\
\cline { 2 - 5 } & Number & Percentage (\%) & Number & Percentage (\%) \\
\hline Pus Discharge POD & 4 & 4.00 & 7 & 7.00 \\
\hline Seroma \& discharge & 3 & 3.00 & 7 & 7.00 \\
\hline Nil & 93 & 93.00 & 86 & 86.00 \\
\hline Total & 100 & 100.00 & 100 & 100.00 \\
\hline
\end{tabular}

\section{Conclusion}

As per the research analysis, one application of chlorhexidine is found to possess better antiseptic properties in preparing surgical site which is found to possess better efficacy and costeffectiveness comparing other antiseptic methods. Alcoholbased solutions are quick, sustained, and sturdy with a broader spectrum of antimicrobial activity. These properties made it ideal for extended open surgeries with the potential for infection or surgical spillage, for percutaneous procedures with indwelling catheters, and for prosthesis implantation when minimizing skin colony counts so as to stop infection from the hardware. When using these products care should be taken in two ways 1) allow adequate drying time and 2) remove excessive hair from the prepared field which can delay the vaporization ability of alcohol. Findings of this study in conjunction with previous study reports of general surgery and gynaecology further support our belief that the utilization of an antisepsis protocol of twentytwo chlorhexidine- the alcohol-based solution should be used as a routine antisepsis protocol for all the procedures.

\section{References}

1. Watanabe A, Kohnoe S, Shimabukuro R et al. Risk factors associated with surgical site infection in upper and lower gastrointestinal surgery. Surg Today 2008;38:404-12.

2. Altemeier WA, Culbertson WR, Hummel RP. Surgical considerations of endogenous infections - sources, types, and methods of control. Surg Clin North Am 1968;48:22740.

3. Centers for Disease Control and Prevention. Nosocomial outbreak of Rhizopus infections associated with Elastoplast wound dressings - Minnesota. MMWR. 1978;27:33-4.

4. Wiley AM, Ha'eri GB. Routes of infection. A study of using "tracer particles" in the orthopaedic operating room. Clin Orthop Relat Res 1979;(139):150-55.

5. Webster J, Osborne S. Preoperative bathing or showering with skin antiseptics to prevent surgical site infection. Cochrane Database of Systematic Reviews 2015, 2. Art. No.: CD004985. DOI: 10.1002/14651858.CD004985.pub5.

6. Johnson AJ, Daley JA, Zywiel MG, Delanois RE, Mont MA. Preoperative chlorhexidine preparation and the incidence of surgical site infections after hip arthroplasty. The Journal of arthroplasty 2010;25(6):98-102.

7. Weinstein RA, Milstone AM, Passaretti CL, Perl TM. Chlorhexidine: expanding the armamentarium for infection control and prevention. Clinical Infectious Diseases. 2008;46(2):274-81.

8. Edmiston Jr CE, Okoli O, Graham MB, Sinski S, Seabrook GR. Evidence for using chlorhexidine gluconate preoperative cleansing to reduce the risk of surgical site infection. AORN journal 2010;92(5):509-18.

9. Levin I, Amer-Alshiek J, Avni A, Lessing JB, Satel A, Almog B. Chlorhexidine and alcohol versus povidoneiodine for antisepsis in gynecological surgery. Journal of Women's Health 2011;20(3):321-4.

10. Menderes G, Ali NA, Aagaard K, Sangi-Haghpeykar H. Chlorhexidine-alcohol compared with povidone-iodine for surgical-site antisepsis in cesarean deliveries. Obstetrics \& Gynecology 2012;120(5):1037-44.

11. Edmiston Jr CE, Seabrook GR, Johnson CP, Paulson DS, Beausoleil CM. Comparative of a new and innovative $2 \%$ chlorhexidine gluconate-impregnated cloth with $4 \%$ chlorhexidine gluconate as topical antiseptic for preparation of the skin prior to surgery. American journal of infection control 2007;35(2):89-96. 
12. Maiwald M, Chan ES. The forgotten role of alcohol: a systematic review and meta-analysis of the clinical efficacy and perceived role of chlorhexidine in skin antisepsis. PloS one 2012;7(9):e44277.

13. Reichman DE, Greenberg JA. Reducing surgical site infections: a review. Reviews in Obstetrics and Gynecology 2009;2(4):212-21

14. Hemani ML, Lepor H. Skin preparation for the prevention of surgical site infection: which agent is best? Reviews in urology 2009;11(4):190.

15. Culligan PJ, Kubik K, Murphy M, Blackwell L, Snyder J. A randomized trial that compared povidone iodine and chlorhexidine as antiseptics for vaginal hysterectomy. American journal of obstetrics and gynecology 2005;192(2):422-5.

16. Sowapat K, Soontrapa S, Sakondhavat C. Preoperative vaginal preparations for abdominal hysterectomy for the prevention of febrile morbidity: savlon douching vs povidone-iodine painting. Journal-medical association of Thailand 2006;89(1):20.

17. Adler MT, Brigger KR, Bishop KD, Mastrobattista JM. Comparison of bactericidal properties of alcohol-based chlorhexidine versus povidone-iodine prior to amniocentesis. American journal of perinatology 2012;29(06):455-8. 